\title{
A REVISED METHOD FOR ESTIMATING REDUCTION OF NATURAL POPULATIONS EFFECTED BY POISONING
}

\author{
RYo TANAKA \\ Zoological Laboratory, Kochi Women's College, Kochi City
}

\section{Introduction}

In the foregoing paper, ${ }^{2)} \mathrm{I}$ presented a method to measure the reduction ratio of natural populations exposed to poison baits following the parabolic formula $y=(x / P)^{\beta}$, which seems likely to widely suit mark-and-release records at least for field mice. Universally, it is at first requisite in the census to make allowance of the amount of the index $\beta$, i. e., whether or not the probability of capture for marked animals is distinct from that for unmarked ones; especially so in comparing populations before and after poisoning treatments since the survived population may usually consist of both previously marked and unmarked individuals, which are supposed to retain their prepoisoning probabilities of capture respectively.

Most recently, however, I have been led to turn to the view that the census method by HAYNE's second equation $\left.{ }^{5}\right) y=(P-x) p$, which was proposed for removal trapping, would be most available for practical works at least concerning field mice for the present. This paper attempts to describe and evaluate a revised procedure of the post-poisoning census founded on the principles of this equation, accompanied by some results with reference to the method executed on the grassland slope of Mt. Fuji.

\section{Census by the maximum likelihood method}

The census method using my formula, although it may fit trapping records, was criticised ${ }^{6 \prime}$ to be unreasonable by H. Sugiyama, ${ }^{*}$ Laboratory of Stochastics, Osaka City Medical College, for reasons that $P$ of the formula, a special term of the polynominal $y=A_{1}+A_{2} x+A_{3} x^{2}+\cdots \cdots$, is difficult to afford substantial foundation for expressing a population size. He, in turn, attempted a population $N$ by the maximum likelihood method on the assumptions that probability of capture $(\pi)$ for marked animals and that $(p)$ for unmarked

\footnotetext{
* I owe him many thanks for his personal advices.
} 
ones are in general different from each other and kept constant and that events of being captured for both members are independent of each other. He induced likelihood $L$ from each daily trapping record of a mark-andrelease operation as follows :

$$
L=\text { const. } \times I I\left\{\pi^{\alpha}(1-\pi)^{\gamma}\right\} \times I I\left\{\left(\begin{array}{c}
N-\lambda \\
\mu
\end{array}\right) p^{\mu}(1-p)^{N-\nu}\right\}
$$

in which, $N, \pi$ and $p$ are unknown parameters, and $\alpha$ is number of captures previously marked, $\gamma$ number of previously marked animals not captured, $\lambda$ total number of unmarked captures untill the day before, $\mu$ number of unmarked captures, and $\nu=\lambda+\mu$ (II: symbol of accumulative product). From this, maximum likelihood estimates $\hat{\pi}$ and $\hat{p}$ are derived,

$$
\hat{\pi}=\frac{\Sigma \alpha}{\Sigma \alpha+\Sigma \gamma} \quad \hat{p}=\frac{\Sigma ' \mu}{\Sigma(\hat{N}-\lambda)}
$$

Here, since $\hat{\pi}$ is determined by the former, if we can get the maximum point of the curve that is formed by the values of $I I\left\{\left(\begin{array}{c}N-\lambda \\ \mu\end{array}\right) p^{\mu}(1-p)^{N-\nu}\right\}^{*}$ plotted on various given values of $N$, the $N$-value equivalent to the maximum is the final estimate of maximum likelihood.

We shall consider the assumptions underlying this method; the constancy of $\pi$ and $p$ during a trapping period is first needed. This corresponds not wholly with my supposition ${ }^{2}$ that the ratio $\pi / p$ is constant although they may shift, drawn from $y=m x /(P+\overline{m-l} x)$, but the last equation is mathematically not expected to hold good with random values of $x$, so that the latter part of my presumption should be rejected, according to Sugryama; moreover it is statistically verified in the data2) of Clethrionomys rufocanus that actual values of $\pi$ and $p$ remain invariable. In this method, therefore, individuals, if any, abnormally developed in trap wariness will be spontaneously excluded from the estimated population, while others higher than normal in trappability might in some way distort the result, thus a precaution must be taken to reject statistically any trapping record showing aberrant probability of capture in the census procedure.

Another assumption that trapping events are independent of each other between marked and unmarked members seems to me to be questionable because one trap after having captured one animal usually shuts out all of the follow-up, the practical effect of the error introduced by this difficulty being not clear. As opposed to this method, the parabolic equation necessitated not always such two assumptions.

* This is denoted as $L / C$ (C: another constant). 
For instance, $N$ of the vole can be estimated by this method from the trapping records ${ }^{2}$ July 26 to August 5 as follows; we get $\hat{\pi}=0.61$ and $\hat{p}=69 /(11 \hat{N}-571)$, from the latter, $p$ being computed against given values of $N$ as

$\begin{array}{cccccccc}N & 92 & 98 & 100 & 102 & 104 & 106 & 112 \\ p & 0.1565 & 0.1361 & 0.1304 & 0.1252 & 0.1204 & 0.1160 & 0.1044 .\end{array}$

The curve of $L / C$, drawn through these sets of values, dependent upon $N$ is shown in Figure 1 which gives the maximum equivalent to $\hat{N}=102$ with $\hat{p}=0.125$.

The population sizes recalculated in the same way from my other data so far obtained are all more or less larger than those estimated relying upon the parabolic formula, $P$ of which, therefore, would be considered as some constant value different from, presumably in some degree smaller than, the true size of population. Regardless of the fact, the meaning1) 2) of $\beta$, the mark-and-release index, is still retained insomuch as its relation to 1 is reflecting whether $\pi$ is superior or inferior to $p$ (the case of $\beta>1$ cannot be statis-

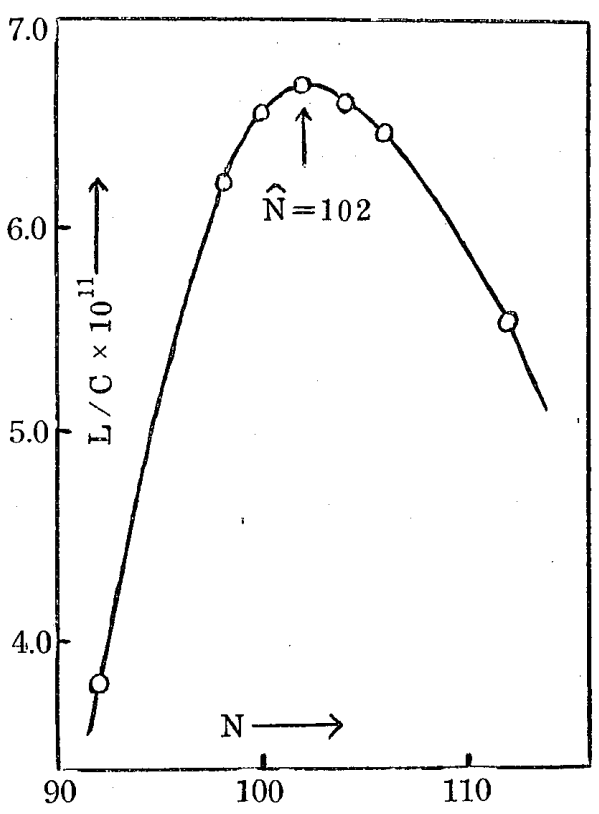

Fig. 1. Curve of $L / C$ dependent upon $N$ in Clethrionomys rufocanus tically verified for the brown rat $^{3}$ ) but the tendency is insured from $\hat{\pi}=0.013<\hat{p}=0.069$ ), whereas $\beta$ has yet failed to be expressed as any function of $\pi$ and $p$.

On the other hand, Sugiyama asserts that expectation of $Y$, probability variate eqivalent to number of unmarked captures, approaches $(N-x) p$ provided with random values of $x$, total number previously marked (or total number of unmarked captures $\lambda$ when any death occurred), namely $E(Y)=$ $(N-x) p$, which agrees with HAYNE's second equation that was regarded by him as consistent also with cases of mark-and-release; there ${ }^{5 /}$ he called no attention to the hetrogeneous trappability, and attached little importance to this equation. A population estimate, thus yielded, must agree with that of maximum likelihood because of the same assumptions underlying both methods, although the former dealing with only events of unmarked members is likely to be more influenced by sampling errors. 
In practical cases, however, a close coincidence between them can be demonstrated in the data of $\mathbf{C l}$. rufocanus ${ }^{2)}$ as well as of $\mathbf{C l}$. smithii ${ }^{1)}$; the former method may produce $N=100$ and $p=0.135$ even when a fitting line is graphically drawn setting more weight at larger values of $x$ in rufocanus, and in the same way it may give $N=78$ and $p=0.083$ against $\hat{N}=76$ and $\hat{p}=$ 0.090 in smithii.

We have thus comprehended that the simple method following $E(Y)=(N-x) p$ may give rise to a reliable conclusion approximating that expected by the method of maximum likelihood which is far more laborious in its procedure; that is the reasons why it is highly appreciable for practical use. Then a revised method for evaluating a post-poisoning population ought to be devised instead of that previously2) suggested by myself.

\section{Reduction ratio of populations}

First, the pre-poisoning population $N_{1}$ is measured either by the maximum likelihood method or by using $Y=(N-x) p$ from trapping records during about 5 to 7 days. It is necessary to determine $\pi$ and $p$ besides $N_{1}$ from the records, $\pi$ being easily computed by $\pi=\Sigma \alpha /(\Sigma \alpha+\Sigma \gamma$ ) or $X=x \pi$ ( $X$ : number of marked captures each day), because both values are presumed to remain the same through the period of poisoning and post-poisoning operations, which is desirable to be as short as possible since much ingress of adjacent residents seems likely to introduce errors in conclusions during the period, but too brief process may make it difficult to expect a good estimate. In practice, it would be adequate to spend a few days in poisoning and 3 to 5 days in after working.

The post-poisoning population $N_{2}$ may be estimated by

$$
N_{2}=\left(N_{1}-M\right) r^{\prime}+M r
$$

where $M$ is the total number of marked and released animals immediately before poisoning, $r$ and $r^{\prime}$ respectively reduction ratio of marked and of unmarked. Here, if $r$ and $\boldsymbol{r}^{\prime}$ can be assessed, $N_{2}$ will be computed, resulting in giving the reduction ratio of the population $R=N_{2} / N_{1}$.

Let numbers of marked and unmarked captures be $X_{1}, Y_{1} ; X_{2}, Y_{2} ; \ldots \ldots \ldots$; $X_{n}, Y_{n}$ respectively on 1 st, $2 \mathrm{nd}, \cdots \cdots \ldots$, nth day after poisoning, with regard to marked captures

$$
\begin{aligned}
& X_{1}=M r \pi \\
& X_{2}=\left(M r+Y_{1}\right)_{\pi}
\end{aligned}
$$




$$
\begin{aligned}
X_{3}= & \left(M r+Y_{1}+Y_{2}\right) \pi \\
& \cdots \cdots \cdots \cdots \cdots \cdots \\
X_{n}= & \left(M r+Y_{1}+Y_{2}+\cdots \cdots \cdots \cdots \cdots+Y_{n-1}\right) \pi,
\end{aligned}
$$

and with regard to unmarked

$$
\begin{aligned}
Y_{1}= & \left(N_{1}-M\right) r^{\prime} p \\
Y_{2}= & \left\{\left(N_{1}-M\right) r^{\prime}-Y_{1}\right\} p \\
& . . \cdots \cdots \cdots \cdots \cdots \\
Y_{n}= & \left\{\left(N_{1}-M\right) r^{\prime}-Y_{1}-Y_{2}-\cdots \cdots \cdots \cdots \cdots \cdots \cdots \cdots \cdots+Y_{n-1}\right\} p .
\end{aligned}
$$

From these equations, $r$ and $r^{\prime}$ can be determined by the least square method setting homogeneous weight at each as follows;

$$
\begin{aligned}
& r=\frac{\sum X-\left\{(n-1) Y_{1}+(n-2) Y_{2}+\cdots \cdots \cdots \cdots+Y_{n-1}\right\} \pi}{n M \pi} \cdots \cdots \cdots \cdots \\
& r^{\prime}=\frac{\sum Y+\left\{(n-1) Y_{1}+(n-2) Y_{2}+\cdots \cdots \cdots \cdots+Y_{n-1}\right\} p}{n\left(N_{1}-M\right) p} \cdots \cdots \cdots \cdots \cdots
\end{aligned}
$$

If the pre-poisoning popualtion is exhaustively marked, we get $r=\Sigma^{\prime} X / n M \pi$ and $R=r$; if $r=r^{\prime}$ is assumed, likewise $R=r$ but $r$ must be computed by equation (2) or (3).

\section{Poisoning work with Microtus-populations}

Field works for censusing before and after poisoning were conducted about ten days in the fall of 1952, with populations of Microtus montebelli on the south-western grassland slope of Mt. Fuji, which is in greater parts afforested with younglings of Chamaecyaris; they have lately been subjected to severe injury by the vole.

Two plots, each covering 2.8 acres, were selected nearly 400 meters apart from each other. The marking and releasing work was made by laying fifty cage traps of wire net, baited with pieces of potato and distributed in gridpattern at intervals of $15 \mathrm{~m}$ as usual; following the preliminary census four to five days, the population was exposed to poisons for two days, during which every day ten poison baits were set at each site of traps, which were closed the while, and the amount of their consumption by voles was enumerated on the following day. Corn kernel, made to absorb red solution of $\mathrm{CH}_{2} \mathrm{FCOONa}$, was used as poison bait; one-third or a half of each grain contains in average the lethal dose of the poison to one vole.

The trapping records (Table 1 ) are not so sufficient as to enable us to count by the present method pertinent values of $\pi$ and $p$, comparable to 

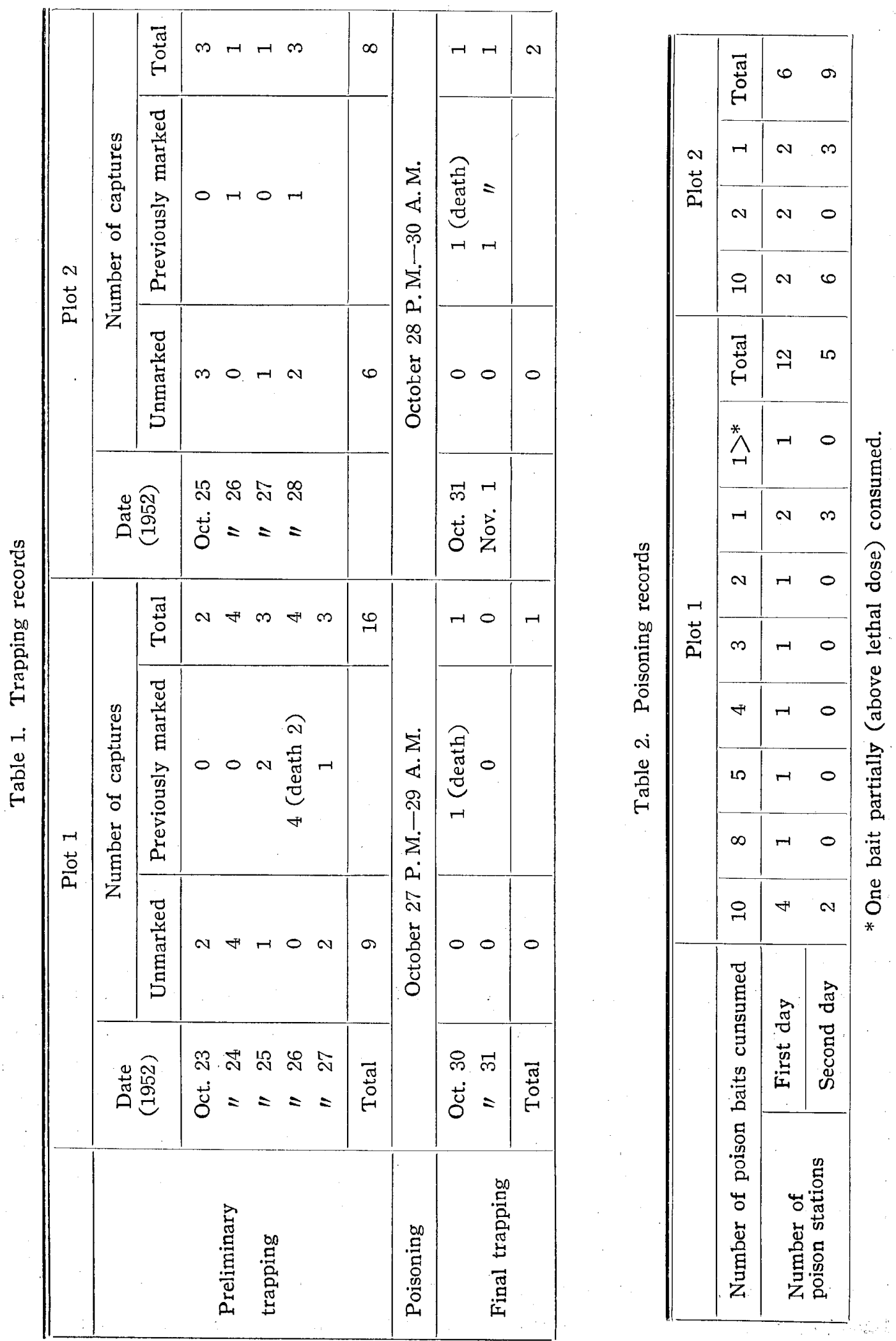
those ( $\hat{\pi}=0.28, \hat{p}=0.06^{*}$ ) previously known of the vole on the grassland riverside of the $R$. Omono, Akita Prefecture, insomuch that it seems rather more reasonable to estimate $N_{1}$ and $N_{2}$ by applying these values to the present data. In this way we account $N_{1}=34$ in plot 1 and 28 in plot 2 from the preliminary records, while, no one but one or two previously marked animals having been captured in the final two-day trapping, we are given $\boldsymbol{r}=\Sigma X / n M \pi, r^{\prime}=0$ and $R=M r / N_{1}$ in this case, thus $R=0.05$ and 0.13 being computed.

By a slight shift of view-point, it is possible to approach crude numbers of kills by taking into consideration the consuming frequency of poison baits caused by voles; the baits were acceptable also to ants, myriapods and. slugs, but the consumption by these animals could easily be distinguished from that by voles, thus extinction of one or more grains from a poison station was doubtlessly referable to the latters; sometimes new burrows or runways were found opened at such stations. In Table 2 is shown the frequency of poison stations, at which various numbers of corn kernels were cosumed by voles.

Let the number of grains consumed at a station be $n$, at least one kill will certainly be effected and as many as $n / 2$ kills would be probably expected under the best condition, considering the behavior of voles for food in view of acute toxicity of the poison palsying them shortly after it is taken. In this way the reasonable limitations of numbers of kills can be given as $17 \sim 44$ in plot 1 and $15 \sim 47$ in plot 2 . If we assume that these kills resulted from the reduction ratio 0.1 above calculated, the initial populations prove to be $19 \sim 49$ and $17 \sim 52$, each average of which approximates respective value of $N_{1}$ in both plots. Anyway, whereas the majority of population seemed to have been killed, it is certain that some voles remained released from death.

Comparing the sites, animals' visits at which are proved by the traps with those proved by the poison baits, it is clear that the latter exceeds the former in the number of the sites as well as in the distribution range; hence we may say that members of the populations are more accessible to poison baits than to traps, that is to say, the "new object reaction" ") exhibited by voles against traps is plainly stronger than to the powerful poison baits. A marked tendency of this vole for lower trappability in contrast with other species has already been acknowledged by us in accordance with its $\beta$-value lesser than 0.5. At large, an important difficulty in the census procedure based on trapping records may consist in such new object reaction of animals

* Recalculated by the maximum likelihood method. 
to traps; especially with the case of the present species it might be emphasized. Therefore, after all, enumeration of kills from the consuming frequency of poison baits would seem practically of greater advantage over estimation of post-poisoning populations from trapping records in such voles as this.

\section{Summary}

1. We have reasons to think that the census method using $E\left(Y^{*}\right)=(N-x) p$ is most highly appreciable in practice for the present, the equation holding on the assumptions that events of capture for marked and unmarked animals are independent of each other and respective probability of the event for both is constant; $P$ of $y=(x / P)^{\beta}$ seems to give some underestimate as compared with $N$, but the index $\beta$ keeping its meaning as before may still characterize general trap response of a population. Thus a revised method for measuring post-poisoning populations was devised on the basis of principles of the former equation.

2. Field works were executed with Microtus-populations to make practical use of this procedure; the reduction ratio of them was computed as about 0.1 by applying previously known values of $P$ and $\pi$ for want of the data, the results approximating round numbers of kills which were on biological grounds determined from the consuming frequency of poison baits. The "new object reaction" of the populations was found plainly smaller to the powerful baits than to traps, accordingly, for this species tending to have lesser trappability, enumeration of kills from the consuming frequency of poisons would seem practically of more advantage over estimation by trapping.

\section{Literature}

1) TANAKA, R. Estimation of vole and mouse populations on Mt. Ishizuchi and on the uplands of southern Shikoku: J. Mamm., 32 (4) : 450-58 (1951)

2) Theoretical justification of the mark-and-release index for small mammals. Bull. Kochi Wom, Coll. 1: 38-47 (1952)

3) — \& F. Kawashima Movement and population of the brown rat in Kochi City. Annot. Zool. Jap., 24 (4): 225-33 (1951)

4) — \& S. TERAmura A Study of a mid-summer population of the Japanese field vole infested with Tsutsugamushi disease. J. Mamm., 34 (3):
345-52 (1953); Japanese summary, Jap. J. Sanit. Zool., $3(3,4): 135$ (1953)

5) HAYne, D. W. Two methods for estimating population form trapping records. J. Mamm., 30(4) : 399-411 (1949)

6) Tanaka, H., H. Sugiyama et al. Stochastical consideration of rats and mice (III), on their populations (Further rcport). Pub. at 5th Ann. Meet. Jap. Soc. Sanit. Zool. (1953)

7) Chitty, D. \& D.A. Kempson Prebaiting small mammals and a new design of live trap. Ecology, 30 (4i) : 536-42 (1949) 